$(\mathrm{p}=0.001)$. Positive correlation between noise exposure and duration of hospitalization was determined. Infants who failed at 1001 and $1501 \mathrm{~Hz}$ had similar Bayley II Infant Development Scale scores and there were no difference between groups.

Conclusion Major noise source in NICU was found to be the incubators. Although hearing loss was not detected in any infants, hearing tests at sixth months of life were adversely affected.

\section{A COMMON PROBLEM FOR NEONATAL INTENSIVE CARE UNIT'S: LATE PRETERM INFANTS}

doi:10.1136/archdischild-2012-302724.1281

if Çelik, 'G Demirel, 'FE Canpolat, 1,2U Dilmen. 'Neonatology, Zekai Tahir Burak Maternity Teaching Hospital; ${ }^{2}$ Pediatrics, Yildirim Beyazit University, Faculty of Medicine, Ankara, Turkey

Background Late preterm infants are physiologically and metabolically immature than term infants, and the incidence of late preterm birth is increasing. These infants have higher risks of medical complications such as respiratory distress, hypoglycemia, hyperbilirubinemia, sepsis, feeding difficulty and poor neurodevelopmental outcome than term infants.

Objective We aimed to evaluate the clinical and demographic characteristics, and short-term outcomes and clinical course of late preterm infants who were admitted to our neonatal intensive care unit (NICU).

Materials and method Data from NICU admissions of 605 late preterm and 1477 term infants in 1 year period between June 2010 and May 2011 were analyzed.

Results Late preterm and total delivery numbers were 2004 and 18854. NICU admission rate of late preterm infants was $30 \%$, respectively. Mean gestational week and birth weight were $35^{1 / 7} \mathrm{~W}$ and $2352 \mathrm{~g}$. Admission diagnosis were respiratory distress (46.5\%), low birth weight $(17.5 \%)$, jaundice $(13.7 \%)$, polycythemia $(8.1 \%)$, hypoglycemia (4\%) and feeding difficulty $(13.1 \%)$, and these morbidities' rates were higher than term infants $(p<0.001)$. During hospital stay; jaundice, polycthemia, hypoglycemia, feeding difficulty, sepsis, apnea and pneumonia rates were 300 (49.6\%), 98 (16.2\%), $88(14.5 \%), 218(36 \%), 85(14 \%), 7(1.2 \%)$ and $27(4.5 \%)$, respectively. Overall mean hospitalization length was $7.5 \pm 9.1$ days. Mortality and rehospitalization rate was $2.1 \%$ and $4.4 \%$, and higher than term infants $(<0.001)$

Conclusion We concluded that late preterm infants should be followed closely for these complications just after birth and preventive strategies should be put in practice.

\section{AN ANALYSIS OF RETINOPATHY OF PREMATURITY REQUIRING TREATMENT OVER 5YEARS PERIOD IN OUR NEONATAL UNIT}

doi:10.1136/archdischild-2012-302724.1282

A Kahlil, AM Murphy, 0 O'Leary, T Stack, C Sreenan, R Philip. Neonatal Intensive Care Unit, Regional Maternity Hospital, Limerick, Ireland

Background Proliferative retinopathy occurs primarily in premature LBW infants as a result of incomplete vasculogensis of the retina at the time of birth. It can be mild, self limiting with no visual defects or progressive leading to blindness.

\section{Screening guidelines}

1. Birth weight $<1.5 \mathrm{Kg}$.

2. Gestational ane $<32$ weeks

3. Birth weight $1.5 \mathrm{kgs}-1.8 \mathrm{kgs}$ and/or Gestational age $32-34$ weeks (if received supplementary oxygen for $\geq 12$ hours.

4. If one twin is in the screening criteria and has eye changes.

Aim To review the number of babies $<1500 \mathrm{~g}$, and or Gestational age of $<32 / 40$ who developed ROP required treatment, focusing on infants $<1 \mathrm{Kg}$.
Methods Retrospective chart review of babies born in the Regional Maternity Hospital Limerick between 1stJan 2007 and 31st Dec 2011 with ROP requiring treatment.

Results During the study period a total of 225 infants with B.wt < $1500 \mathrm{~g}$ and/or G.A < 32/40 were admitted to the neonatal unit. All these infants were screened for ROP as per unit guidelines. $93.3 \%$ $(\mathrm{N}=210)$ infants were $<32 / 40$, and $83 \%(\mathrm{~N}=187)<1500 \mathrm{~g}$, of these $28 \%(\mathrm{~N}=64)$ infants weighed less than $1 \mathrm{Kg}$.

There were 10 infants (4.4\%) who developed ROP requiring treatment, 9 of these infants were in the ELBW category, the tenth baby was only 40gs over the criteria for VLBW(B.wt 1040gs).

All 10 babies have disease in zone 2.7 stage2. 2 stage 1 and one had stage 4 .

Conclusion In our unit 10 infants(4.4\%)received treatment for ROP over the 5 years period 2007-2011. Our figures are comparable to those reported by Vermont Oxford Network database 2010.

\section{MONITORING THE EFFECT OF NEUROMUSCULAR BLOCKADE IN NEONATES: CURRENT PRACTICE IN THE UNITED KINGDOM}

doi:10.1136/archdischild-2012-302724.1283

M Honsel, C Giugni, J Brierley. Paediatric \& Neonatal Intensive Care Unit, Great Ormond Street Hospital for Children, London, UK

Background and Aims Neuromuscular blocking agents (NMBAs), either intermittent boluses or continuous infusions, are used in infants to facilitate difficult ventilation and lower pulmonary pressures by preventing infant-ventilator asynchrony in e.g. severe meconium aspiration syndrome, persistent pulmonary hypertension or air leak.

Whilst consensus statements and accepted standards regarding NMBA use and assessment exist in adult and paediatric ICU, there exists limited information in NICU, specifically whether clinical assessment, NMBA-monitoring (train-of-4) or formal acceleromyography is optimal. We wanted to ascertain current NMBA monitoring in UK NICU.

Methods Literature search for NMBA assessment in infants and telephone survey of all tertiary NICUs in England, and major units in Wales, Scotland and Northern Ireland, in which we asked the nurse in charge (to ascertain actual rather than perceived optimal practice) about existing protocols, methods used for NMBA monitoring (clinical observation, TOF/acceleromyography) and the use of 'drug holidays'.

Results No standards, or peer-reviewed NMBA guidelines were found. Of 56 units contacted, 2 did not share information and 3 use intermittent boluses of NMBAs rather than continuous infusion. Of the remaining units all $(100 \%)$ clinically assess the patient $1(1.96 \%)$ has a protocol in place, $11(21.57 \%)$ perform regular NMBA-holidays to assess effect and 1 (1.96\%) uses train-of-4 if a patient on NMBA-holiday does not move after 6 hours.

Conclusions We found no peer reviewed NICU-NMBA standards or guidelines in the literature, Only 1 UK unit has any protocol for NMBA assessment. Guidelines/standards for NMBA use in infants need to be urgently introduced.

\section{A NETWORK PERSPECTIVE OF THE MAJOR OUTCOMES OF PREMATURE BABIES LESS THAN $31^{+0}$ WEEKS GESTATION USING A UNIFIED ELECTRONIC SYSTEM}

doi:10.1136/archdischild-2012-302724.1284

'SI Aslam, $2,3 \mathrm{SV}$ Rasiah, ${ }^{3} \mathrm{M}$ Passant. ${ }^{1}$ Neonates, New Cross Hospital, Wolverhampton; ${ }^{2}$ Neonates; Birmingham Women's Hospital NHS Foundation Trust; ${ }^{3}$ Neonates, South West Midlands Newborn Network, Birmingham, UK

Background The Badger electronic system collects data in a standardized manner and allows us to audit the major outcomes of the babies managed within the network. 